\title{
Chronic respiratory symptoms but normal lung function: substantial disease burden but little evidence to inform practice
}

\author{
Milo A. Puhan (D) \\ Affiliation: Dept of Epidemiology, Epidemiology, Biostatistics and Prevention Institute, University of Zurich, \\ Zurich, Switzerland. \\ Correspondence: Milo A. Puhan, Dept of Epidemiology, Epidemiology, Biostatistics and Prevention Institute, \\ University of Zurich, Switzerland, Hirschengraben 84, 8001 Zurich, Switzerland. E-mail: miloalan.puhanduzh.ch
}

@ERSpublications

The disease burden from chronic respiratory symptoms in individuals without airflow obstruction is substantial but evidence to inform practice is largely lacking http://bit.ly/2yFeVHN

Cite this article as: Puhan MA. Chronic respiratory symptoms but normal lung function: substantial disease burden but little evidence to inform practice. Eur Respir J 2019; 54: 1901363 [https://doi.org/ 10.1183/13993003.01363-2019].

The treatment of symptomatic patients with chronic airway obstruction (defined by a forced expiratory volume in $1 \mathrm{~s}$ to forced vital capacity ratio of $<0.7$ ) is widely established. It includes drug and non-drug treatments to slow down disease progression, reduce symptoms and reduce the risk of exacerbations [1-5]. Individuals with chronic airway obstruction but no respiratory symptoms are commonly advised to take preventive measures (e.g. smoking cessation) to slow down the decline of lung function [6]. But there is a third group of individuals that is much more difficult to deal with: individuals who have chronic respiratory symptoms but normal lung function.

The Global Initiative for Chronic Obstructive Lung Disease (GOLD) once defined a stage 0 where lung function is normal but where individuals have cough or phlegm and are thus considered at risk for developing COPD, in particular if they had exposures like smoking, or environmental or occupational hazards. GOLD dropped stage 0 because it predicted too poorly those who progress to airway obstruction [7]. Of course, if there is an exposure like smoking, individuals should receive support to stop smoking. However, there is currently no recommendation on what treatment to offer them if they suffer from chronic respiratory symptoms but do not fulfil the diagnostic criteria for COPD or asthma [8]. Primary care physicians, who see most of these individuals, will consider other differential diagnoses for the rather unspecific symptoms of breathlessness, mucus hypersecretion, wheezing and cough, but are left without recommendations on what to offer patients if no airway obstruction or differential diagnosis is found.

How large is the group of individuals with chronic respiratory symptoms but normal lung function, and do these symptoms have prognostic relevance? A prevalence study conducted in Sweden almost 20 years ago already showed that around $40 \%$ of patients in primary care have at least one chronic respiratory symptom [9]. Even if asthma and COPD was underdiagnosed in that study (around 10\% prevalence combined), it still left a large group of patients with chronic respiratory symptoms but no airway obstruction. A recent American Thoracic Society/European Respiratory Society statement suggested studying the disease burden of these 
individuals by comparing outcomes between symptomatic individuals with and without established chronic airway obstruction [10]. A number of studies have shown that chronic respiratory symptoms are associated with increased risk of mortality in individuals with normal lung function [8]. In studies using computed tomography, individuals with chronic respiratory symptoms but normal lung function had clear radiological evidence of lung disease [8]. Hence, there are individuals with respiratory symptoms who show a number of manifestations typical for COPD where the only reason not to diagnose COPD is normal lung function.

An analysis of the Copenhagen General Population Study by ÇOLAK et al. [11] reported in this issue of the European Respiratory Journal adds important evidence that is particularly relevant for public health and primary care. It provides a population-based estimate for the prevalence of individuals with chronic respiratory symptoms but normal lung function, as well as estimates for their risks for respiratory exacerbations, pneumonia and respiratory death. The analysis included 97955 persons in whom pre-bronchodilator spirometry was available. Respiratory symptoms were ascertained at baseline and almost complete follow-up data was available (thanks to national registries). $7 \%$ had airway obstruction without chronic respiratory symptoms, $7 \%$ had airway obstruction with chronic respiratory symptoms and $54 \%$ had normal lung function and no chronic respiratory symptoms.

An astonishingly large group of $32 \%$ had chronic respiratory symptoms and normal lung function. In this group, the risk for respiratory exacerbations requiring a hospital admission, pneumonia, respiratory and all-cause death was increased by up to $62 \%$ in fully adjusted models compared to individuals with normal lung function and no chronic respiratory symptoms. Risk increases for these outcomes were, as expected, lower than in individuals with chronic airway obstruction and chronic respiratory symptoms, but they were similar or larger than in individuals with chronic airway obstruction but no chronic respiratory symptoms. Importantly, the results were similar for never and current or former smokers. It was not possible to determine if the risk was truly increased due to a respiratory disease and whether such respiratory disease is an early form of COPD or some other respiratory disease. The consistency of results across respiratory-specific outcomes and the clear dose-response relationship with number of chronic respiratory symptoms and higher outcome risks suggest an underlying respiratory disease in at least a substantial proportion of these individuals.

The study by ÇOLAK et al. [11] has a number of characteristics that makes it particularly relevant for public health and primary care. The estimates for the prevalence of individuals with chronic respiratory symptoms and normal lung function and their risks for adverse outcomes are likely to be internally and externally valid. The study is population-based with a response rate of $43 \%$ of randomly selected participants, there is almost complete follow-up and the data allowed for quite extensive adjustment of confounders. Although spirometry was performed without bronchodilation, which may be seen as a limitation, it is common in primary care settings to only have pre-bronchodilator data. Pre-bronchodilator lung function is not sufficiently accurate to diagnose COPD but to identify patients for further diagnostic work-up [12]. Also, chronic respiratory symptoms were assessed by simple questions, which can also be asked in a primary care practice. Another strength is the, on average, almost 9 years of follow-up for the outcomes, which allowed capturing many person-years of follow-up and outcome events.

The results of this study have implications for both research and practice. It is important to find out what type of underlying respiratory disease individuals with chronic respiratory symptoms and normal lung function have and if some individuals still have another differential diagnosis mimicking chronic lung disease. Also, trials like RETHINC are needed to investigate the effects of drug (e.g. long-acting bronchodilators) and non-drug treatments (e.g. pulmonary rehabilitation) for this group of patients [13]. For primary care practitioners, it is important to note that, even in the absence of chronic airway obstruction and another diagnosis, patients with chronic respiratory symptoms and normal lung function are likely to have a respiratory disease with increased risk for exacerbations, pneumonia and early (respiratory) death. As ÇOLAK et al. [11] suggest, it is wise to repeat lung function testing (with bronchodilation) in these patients and to offer preventive measures like smoking cessation. Since it is difficult to provide recommendations for drug and non-drug treatments to lower symptom burden and risk of adverse health outcomes at this point, guideline developers for primary care and respiratory medicine may consider developing recommendations on the management of these patients even if the evidence base for or against available treatments is still very weak.

Conflict of interest: M.A. Puhan has nothing to disclose.

\section{References}

1 Wedzicha JA, Calverley PMA, Albert RK, et al. Prevention of COPD exacerbations: a European Respiratory Society/American Thoracic Society guideline. Eur Respir J 2017; 50: 1602265. 
2 Wedzicha JA, Miravitlles M, Hurst JR, et al. Management of COPD exacerbations: a European Respiratory Society/American Thoracic Society guideline. Eur Respir J 2017; 49: 1600791.

3 Rochester CL, Vogiatzis I, Holland AE, et al. An official American Thoracic Society/European Respiratory Society policy statement: enhancing implementation, use, and delivery of pulmonary rehabilitation. Am J Respir Crit Care Med 2015; 192: 1373-1386.

4 Watz H, Pitta F, Rochester CL, et al. An official European Respiratory Society statement on physical activity in COPD. Eur Respir J 2014; 44: 1521-1537.

5 Press VG, Au DH, Feemster LC, et al. Reducing chronic obstructive pulmonary disease hospital readmissions: an official American Thoracic Society workshop report. Ann Am Thorac Soc 2019; 16: 161-170.

6 Jiménez-Ruiz CA, Andreas S, Lewis KE, et al. Statement on smoking cessation in COPD and other pulmonary diseases and in smokers with comorbidities who find it difficult to quit. Eur Respir J 2015; 46: 61-79.

7 Rabe KF, Hurd S, Anzueto A, et al. Global strategy for the diagnosis, management, and prevention of chronic obstructive pulmonary disease: GOLD executive summary. Am J Respir Crit Care Med 2007; 176: 532-555.

8 Rodriguez-Roisin R, Han MK, Vestbo J, et al. Chronic respiratory symptoms with normal spirometry a reliable clinical entity? Am J Respir Crit Care Med 2017; 195: 17-22.

9 Hasselgren M, Arne M, Lindahl A, et al. Estimated prevalences of respiratory symptoms, asthma and chronic obstructive pulmonary disease related to detection rate in primary health care. Scand J Prim Health Care 2001; 19: 54-57.

10 Celli BR, Decramer M, Wedzicha JA, et al. An Official American Thoracic Society/European Respiratory Society Statement: research questions in chronic obstructive pulmonary disease. Eur Respir J 2015; 45: 879-905.

11 Çolak Y, Nordestgaard BG, Vestbo J, et al. Prognostic significance of chronic respiratory symptoms in individuals with normal spirometry. Eur Respir J 2019; 54: 1900734.

12 Van Den Bemt L, Wouters BCW, Grootens J, et al. Diagnostic accuracy of pre-bronchodilator FEV1/FEV6 from microspirometry to detect airflow obstruction in primary care: a randomised cross-sectional study. NPJ Prim Care Respir Med 2014; 24: 14033.

13 Han M. RETHINC: REdefining THerapy In Early COPD for the Pulmonary Trials Cooperative. https:// clinicaltrials.gov/ct2/show/NCT02867761 Date last updated: 26 July 2019. 